\title{
Development of a Computational Fluid Dynamics Simulation Model for a Horizontal Axis Tidal Stream Turbine
}

\author{
C. Fung ${ }^{1}$, R. Howell ${ }^{2}$ \\ ${ }^{1,2}$ Department of Mechanical Engineering, Faculty of Engineering, University of Sheffield. \\ ${ }^{1}$ E-Futures Doctoral Training Centre, University of Sheffield.
}

\begin{abstract}
Tidal stream turbines have generated significant research interests due to their major advantages over some other forms of renewable generation. As this technology is relatively new, deployment of tidal turbines is hindered by various problems. Tidal turbines see a non-uniform incoming flow in most situations. This causes differences in power generation and loading at different positions in the rotation of a turbine blade, which affects turbine performance and downstream fluid dynamics. This paper shows the development of a computational fluid dynamics (CFD) model which is currently being used to study such effects. Discussions on the effect of node counts and clustering on mesh independence, along with a study identifying the appropriate turbulence models for the corresponding flow condition will be covered. Findings were validated with experimental data, and used to construct a complete CFD model which is currently being used to study the effect of a non-uniform velocity profile over turbine performance, loading on turbine blades, and wake.
\end{abstract}

Keywords Tidal Energy; Tidal Stream Turbine; CFD, Fluid Dynamics

1. INTRODUCTION

With the challenging deadline set by the UK government, which aims for an $80 \%$ reduction in greenhouse gas emissions by 2050, much effort has been put into developing and installing renewable technologies in recent years. Due to the predictable nature of tides and a relatively high power generation per unit area of occupancy, tide farms may be advantageous compared to wind farms, PV panels, and other tidal technologies [1].

The basic mechanism of tidal stream turbines is similar to that of wind turbines; however there are various difficulties which are specific to tidal turbines. One prominent problem is that due to the restricted locations of deployment, incoming flow with a non-uniform velocity profile is almost unavoidable [2]. This causes uneven power generation and loading at different phases through a turbine blade rotation, hence affecting turbine performance and downstream flow characteristics. To study this phenomenon, a Computational Fluid Dynamics (CFD) model was developed. The development of this model will be explained in the following sections.

\section{Methodology}

The computational model was built upon an existing turbine designed by Bahaj et al. [3]. This turbine was chosen as it has sufficient corrected experimental data to be used for validation.

Prior to the construction of the 3D model, a series of 2D studies were conducted in order to investigate mesh independence and to identify the appropriate turbulence model to be used. These studies were initially completed on the NACA 0012 aerofoil section under the same flow conditions, as experimental data at the corresponding Reynolds number of a similar aerofoil (NACA0018) are available to be used as references [4].
Findings from these studies were used to construct the 3D CFD model. As the free stream velocity is uniform in this study, only $1 / 3$ of the model is required when periodic boundaries are applied. For validation, the predicted power coefficients $\left(C_{P}\right)$ of the turbine at a range of Tip Speed Ratios (TSR) were compared with that of the experimental results.

\section{RESULTS AND DISCUSSIONS}

\subsection{EFFECTS OF VARYING NODE COUNT AND CLUSTERING}

As expected, in the case when nodes were spread over the aerofoil evenly, the difference between two consecutive data points in both lift and drag measurement decreases when node count increases. As seen in Fig. 1a, these differences in lift became insignificant when the node count reached 400 , meaning that the mesh has reached mesh independence.

Results also showed that by clustering nodes towards the leading and trailing edges, fewer nodes were required to achieve a similar level of accuracy. It can be seen from Fig. $1 \mathrm{~b}$ that a grid with 200 nodes will achieve a higher level of accuracy as compared to a grid with 400 evenly distributed nodes. Despite this, there is an optimal point of clustering: when too many nodes are clustered at both edges, there will be insufficient nodes to cover the centre part of the aerofoil, thereby leading to a decrease in accuracy. 

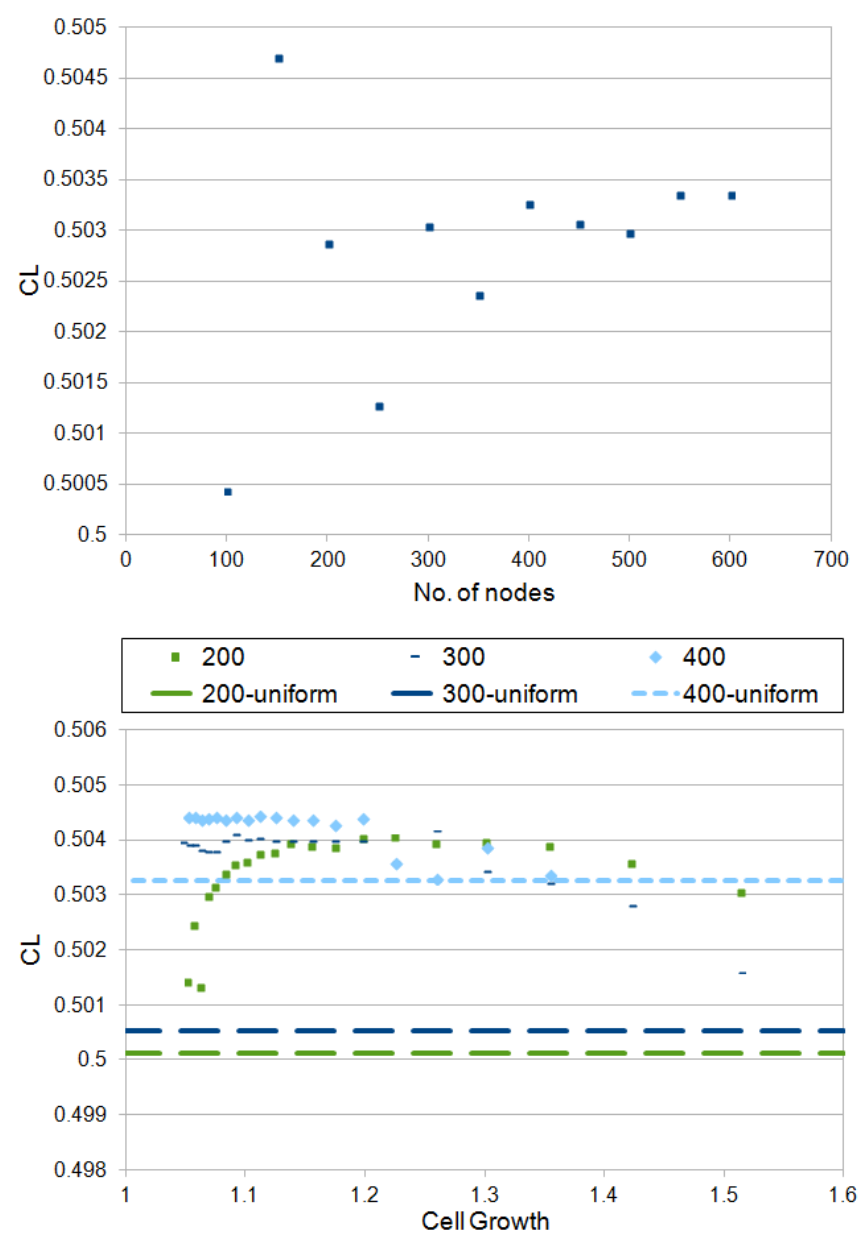

Figure 5(a). (Top) Lift vs. node counts; (b). (Bottom) Effect of node clustering on lift. Increasing cell growth indicates the a grid with less clustered nodes around both the leading and trailing edges.

\subsection{Selection of tURBUlence model}

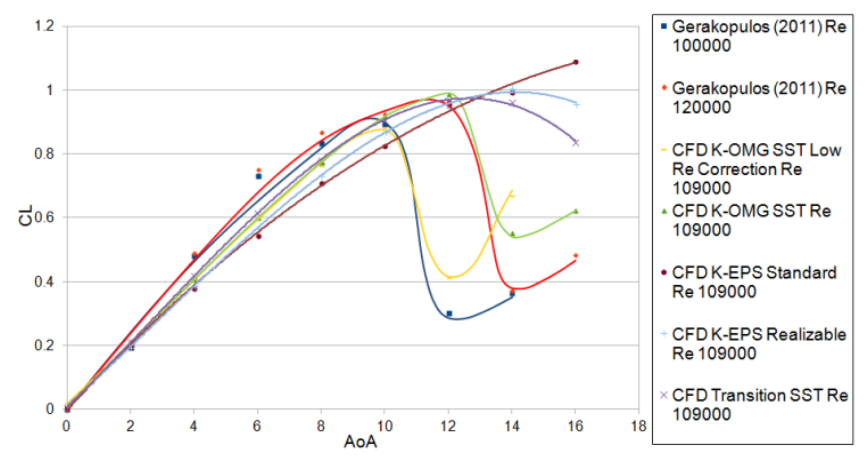

Figure 6. Lift predicted by different turbulent models, compared with experimental data of a similar aerofoil.

Comparing with experimental data, it can be seen from Fig. 2 that the K- $\omega$ SST models gave the closest prediction with respect to the aerofoil's lift. Whether the model should be used with extra damping function for low Reynolds number flow depends on the Reynolds number.

\subsection{D CFD MODEL}

Comparison between experimental data and predictions made by the CFD model on turbine power at different TSR is shown on Fig. 3. Despite the differences between the two sets of data, the overall trends have similar characteristics.
The accuracy of the CFD model will be sufficient for future studies.

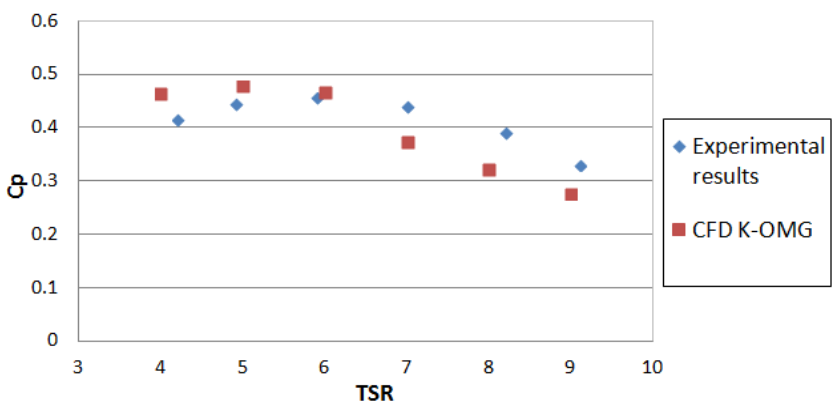

Figure 7. Comparison on power between experimental data and CFD predictions.

\section{CONCLUSIONS}

A CFD model with sufficient level of accuracy and computational economy was created specifically for a tidal turbine. Methodology and findings of individual studies were also discussed. A complete tidal turbine CFD model is currently being used to study the effect of a non-uniform water velocity profile approaching the turbine on its performance, the loading on turbine blades, and the characteristics of the wake.

\section{ACKNOWLEDGEMENTS}

This study was conducted as part of a PhD programme. The author would like to thank ESPRC for funding this project via E-Futures Doctoral Training Centre.

\section{REFERENCES}

1. MacKay D. Sustainable Energy - without the Hot Air. 1st ed. Cambridge: UIT; 2008.

2. Bryden I, Couch S, Owen A, Melville G. Tidal Current Resource Assessment, 2006; 221: p. 125-135

3. Bahaj A, Molland A, Chaplin J, Batten W. Power and Thrust Measurements of Marine Current Turbines under Various Hydrodynamic Flow Conditions in a Cavitation Tunnel and a Towing Tank, 2007; 32: p. 407-426.

4. Gerakopulos R. Investigating Flow over an Airfoil at Low Reynolds Numbers Using Novel Time-Resolved Surface Pressure Measurements. MSc. Waterloo, University of Waterloo; 2011. 